\title{
Correspondence
}

\section{Are their spectacles really necessary?}

SIR, This editorial,' which is overtly critical of opticians, unjustifiably so in my view, has been brought to my attention. Although I am writing in my private capacity as an experienced ophthalmic optician, I am also the Scottish representative of the British College of Ophthalmic Opticians and chairman of the National Optical Consultative Committee.

The main defect in your editorial lies in the assumption that ' $80-90 \%$ of the prescriptions' for the 516 wearers of supposedly unnecessary spectacles out of 13800 school children aged 10 years or less 'have been issued by nonmedically qualified persons.' You give no source for that figure of $80-90 \%$ but it presumably applies to all age groups in the population.

But the 'School Eye Service' is staffed almost entirely by medically qualified ophthalmic practitioners who very probably account for a much higher proportion than 20 to $10 \%$ of refractions in the age group 10 years or under.

I have no access to figures in the Bristol area where Dr Stewart-Brown's survey was done ${ }^{2}$ but I personally have ascertained information about all spectacle dispensing in the Lothian, Scotland, area in February 1985. That month was chosen because it was probably an average month and because after 1 April 1985 these figures will not be available. Of the 85 children under the age of 10 years who had spectacles dispensed 37 were seen by an ophthalmic optician and 48 by a medically qualified ophthalmologist. I suspect the same would be true of the Bristol area, in which case a high proportion of the unnecessary spectacles, if indeed they were unnecessary, were prescribed by ophthalmologists.

Both Dr Stewart-Brown's paper, and indeed your own editorial, admit that hypermetropia requiring spectacles can well exist in the presence of normal visual acuity, yet these cases may constitute a significant proportion of those who received unnecessary spectacles.

Your editorial directs attention to a possible tendency for spectacles to be prescribed for headaches. The belief is widespread among ophthalmologists as well as general medical practitioners that even minor refractive errors are a cause of headaches which can be cured by spectacles.

Dr Stewart-Brown admits ( $p$ 879) that a proportion of children who appear to have normal visual acuity may have been inadequately tested by a series of medical officers with variable training (i.e., non-specialists, presumably).

You are critical of a 'non-medically qualified person' converting 'what begins as a simple task, a refraction,' into a 'pseudo-medical event.' In rebuttal I would claim that the main contributor to the early diagnosis of glaucoma as well as cataract and other diseases (mainly ophthalmic but some general) in this country is the ophthalmic optician. 30 Elder Street, Edinburgh

JACK BROWN

\section{References}

1 Editorial: Are their spectacles really necessary? Br J Ophthalmol 1985; 69: 873.
2 Stewart-Brown S. Spectacle prescribing among 10-year-old children. Br J Ophthalmol 1985; 69: 874-80.

**T The figure for ' $80-90 \%$ of the prescriptions' being issued by opticians was an estimate based on information issued by the Federation of Optical Corporate Bodies, 22 Nottingham Place, London W1, 'Optics at a Glance' 1984. This document states that $86.7 \%$ in 1983 were carried out by opticians and $67 \%$ of these tests resulted in a prescription for spectacles. Mr Brown appears to think that Dr StewartBrown's survey refers only to the Bristol area. However, if he consults the original paper again he will see that the children studied were all those born in the United Kingdom (excluding Northern Ireland) in the one week 7-11 April 1970.-Editor, $B J O$.

\section{Transient monocular obscuration- amaurosis fugax?}

SIR, I was very much interested in the paper 'Transient monocular obscuration-? amaurosis fugax: a case report' by Kosmorsky, Rosenfeld, and Burdel. ${ }^{1}$ This was the case of a 73-year-old hypertensive man five years after right cataract extraction with iris fixation lens implant intermittently treated with pilocarpine to stabilise the lens. He presented repeated visual blurring the cause of which was found to be bleeding from abnormal superior angle vessels.

In 1972 we published the case of a 58-year-old hypertensive woman ${ }^{2}$ who presented spontaneous hyphaema after topical administration of phenylephrine $10 \%$ for routine funduscopy followed by repeated pilocarpine $2 \%$ in order to speed the reconstriction of the pupils. A blood clot was suspended at 12 o'clock of the pupillary border, but we could not observe any abnormal vessel in the area. We believed that perhaps a previously present abnormal vessel was pressed upon by the fibres of the pupillary sphincter in rapid contraction caused by pilocarpine and then ruptured, causing the bleeding; however, no evidence of the ruptured vessel could be found afterwards. Fechner ${ }^{3}$ described a case of spontaneous hyphaema with a ruptured microaneurysm at the pupillary margin and quoted a similar case described in 1932 by Tyson which was accompanied by vascular abnormality in one occipital lobe. Our patient, ${ }^{2}$ who presented in 1976, has not had a recurrence of spontaneous hyphaema since then.

I am wondering if on the background of fragile new formed vessels near the iridectomy in the patient described by Kosmorsky et al. the intermittent administration of pilocarpine did not also play a role (perhaps by iris 'gymnastics' or by some pharmacological properties) in the appearance of hyphaema.

The differential diagnosis from carotid insufficiency was suggested by us in 1975 in a case of a 47 -year-old woman ${ }^{4}$ with anomalous iris vessels in the right eye, right orbit cavernous haemangioma, and a seven-year period of repeated attacks of intermittent blurring of vision due to spontaneous hyphaema. After the excision of the orbital haemiangioma in 1973 there have been no more attacks of spontaneous hyphaema up to the present.

Owing to the rarity of cases of spontaneous hyphaema I 
believe the article published in your journal this year to be important; it serves as a warning of the less known aetiological possibilities of intermittent blurring of vision. Moreover the symptom of erythropsia seems to me very important and contributory to the correct diagnosis.

Neuro-ophthalmic Unit,

Department of Ophthalmology,

Beilinson Medical Center,

Petah Tiqva 49 100, Israel

\section{References}

1 Kosmorsky GS, Rosenfeld SI, Burde RM. Transient monocular obscuration-? amaurosis fugax: a case report. Br J Ophthalmol 1985; 69: 688-90.

2 Manor RS, Sachs W. Spontaneous hyphema and vessel anomaly. Arch Ophthalmol 1975; 93: 1056-8.

3 Fechner PV. Spontaneous hyphaema with abnormal iris vessels. Br J Ophthalmol 1958; 42: 311-5.

4 Savir H, Manor RS. Spontaneous hyphema and vessel anomaly. Arch Ophthalmol 1975; 93: 1056-8.

SIR, Any response to the query by Dr Manor about the possible association of pilocarpine usage and the occurrence of hyphaema in a group of such patients must be purely conjectural. Pilocarpine increases permeability and increases vasodilatation of iris vessels, ${ }^{12}$ at least in experimental animals. If the new vessels in these patients were really fragile, one might suppose that there could be increased diapedesis of red blood cells and thus hyphaema, but the intermittency bespeaks a purely pharmacological effect. If pilocarpine is to be indicted, it would be more logical to suggest a mechanical stretching of vessels secondary to the effect of pilocarpine on the pupillary sphincter. On the other hand spontaneous bleeding from 'aneurysms of the iris' has been reported in a patient without any past surgical disorder. ${ }^{3}$

RONALD M BURDE

Departments of Ophthalmology and

Neurology and Neurological Surgery,

Washington University School of Medicine,

St Louis, Missouri 63110,

USA

\section{References}

1 Stocker FW. Experimental studies on the blood-aqueous barrier. Arch Ophthalmol 1947; 37: 583-90.

2 Swan KC, Hart WM. A comparative study of the effects of mecholyl, doryl, eserine, pilocarpine, atropine, and epinephrine on the blood-aqueous barrier. Am J Ophthalmol 1940; 23: 1311-9.

3 Dewar HA, Manson N. Recurrent monocular blindness. Lancet 1968 ; i: 533 .

\section{Spectacle prescribing among 10-year-old children}

SIR, We enjoyed Stewart-Brown's recent $\operatorname{article~}^{1}$ and the associated editorial ${ }^{2}$ on spectacle lens prescribing among 10year-old children. We note the suggestion that spectacles may have been unnecessarily supplied to many children whose unaided visual acuity was apparently normal. Clearly any evidence for substantial overprescribing in any branch of the National Health Service deserves investigation, though Stewart-Brown rightly recognises the difficulty of assessing the extent of children's refractive problems on the basis of visual acuity measurements alone, particularly when these are made by a variety of personnel under necessarily ill-controlled conditions of measurement. It is of interest to note that this type of controversy is not new, since Donders ${ }^{3}$ was, over a century ago, already discussing the desirability of prescribing spectacles for hypermetropes with asthenopic symptoms.

It seems to us, however, that other important issues besides possible overprescribing arise from the data presented by Stewart-Brown. Her Tables 1 and 3 show that, for example, at least $10 \%$ of those children with 'bilateral marked distant defect' (i.e., both monocular Snellen acuities worse than $6 / 24), 80 \%$ of those with 'bilateral near defect' (worse than 9 on the Sheridan Gardner test), and 7\% of those with 'bilateral marked mixed defect' (worse than $6 / 24$ in each eye at distance and worse than 9 in one eye at near) were unprovided with spectacles. These figures correspond to a total of about 200 children who, in a group that had probably received rather more regular and careful medical examinations than the bulk of their peers, still had possible marked deficiencies in vision without apparently having received refractive assistance. Although the acknowledged limitations of the data do not allow us to assert that the visual abilities of these children would necessarily have been usefully improved by spectacles, it seems reasonable to suggest that children whose visual needs remain unsatisfied may represent at least as disturbing a problem as the approximately equal number whose acuities appear normal and yet have received spectacles.

Department of Ophthalmic Optics,

W N CHARMAN

UMIST,

Manchester M60 1QD

\section{References}

1 Stewart-Brown S. Spectacle prescribing among 10-year-old children. Br J Ophthalmol 1985; 69: 874-80.

2 Smith RJH. Editorial: Are their spectacles really necessary? Br J Ophthalmol 1985; 69: 873.

3 Donders FC. On the anomalies of accommodation and refraction of the eye. London: New Sydenham Society, 1864: chapter 23.

SIR, Drs Charman and Jennings make an important point about the 1970 birth cohort data, presented in my paper on spectacle prescribing. There were indeed a group of children who would appear by available criteria to warrant spectacles who had not been prescribed them, and the discussion did not focus on these children.

They raise a question mark about two quite different groups of children. Those with a distant visual acuity of $\leq 6 / 24$ in both eyes (categories 4 and 10) and those with a near acuity of $\leq 9$ in both eyes with perfect distant vision (category 5).

In the first group there were between 19 and 22 children without spectacles. For some of these children there were legitimate reasons for this lack of spectacles. For example, 